\title{
Positive reinforcement: A test of the Premack theory'
}

Two human Ss were tested on a key pressing task in which two keys produced the same reinforcer, counter points, but on different reinforcement schedules. The results of a contingency arranged between the two keys supported Premack's reinforcement theory; not only do high rate responses reinforce low rate responses, but they do so even when bot:t responses depend on the same reinforcer

The generalization that, for any pair of responses, the independently more probable response will reinforce the less probable one represents the basic assumption of a recent reinforcement theory (Premack, 1959, 1961, 1965). A test of the theory requires first, that the rates of the two responses be determined in a noncontingent period in which both resprnses are simultaneously and freely available to the $\mathrm{Ss}$, and, second, that a contingency be arranged in which the opportunity to engage in the high rate response is dependent upon the occurrence of the low rate response. Previous tests of the theory on rats have shown that the generalization accounts for the reinforcement of bar pressing by drinking, running by drinking, and drinking by running (Premack, 1961, 1962; Schaeffer, 1965). In monkeys, the theory accounts for differential contingent rates of lever manipulation (Premack, 1963). In humans, the generalization holds for the reinforcement of eating by pinball playing, and pinball playing by eating (Premack, 1959).

In each of the preceding tests of the theory, the behavioral topography and consequences of the two responses differed markedly, viz, , bar pressing and drinking, running and drinking, ratchets and plungers, eating and pinball playing. Will the theory also hold for two identical responses, each of which produces the same reinforcing event, but under different schedule requirements? The present study tested this question.

Method

The Ss were a male undergraduate and a female undergraduate enrolled in an introductory psychology class. Both Ss were 19 years old. They were selected on the basis of volunteering to report at the same time daily for unpaid $10 \mathrm{~min}$. test sessions over three consecutive weeks.

The apparatus consisted of a Bud CU-2107 MiniBox in which a Sodeco counter was mounted between two Switchcraft type 60324 telephone keys. One key was programmed on CRF; the other was programmed on FR5. Under the appropriate conditions each depression of the CRF key produced one point on the S's counter, and five depressions of the FR5 key produced one point on the S's counter. A large soundproof test room isolated the Ss from the programming equipment in the adjacent control room where each CRF and FR key depression and total points that appeared on S's counter were recorded.

On the first test day the Ss were conducted into the test room, shown the test box, and given the following instructions: "This is a test of motor coordination. Your job is to try to get as many points as possible in the next $10 \mathrm{~min}$. Begin when you are ready." The $\mathrm{E}$ then left the room, returned in $10 \mathrm{~min}$., dismissed $\mathrm{S}$, and requested that $S$ return at the same time the next day. No further instructions were given over the remaining 16 test sessions.

Each $\mathrm{S}$ was carried through the following five conditions: six days during which both keys were simultaneously and freely available and produced points on their respective schedules (i.e., paired operant sessions); four days during which the CRF key was placed on extinction; two days during which the FR5 key was placed on extinction; four days of the contingent case; and finally, one day on which the Ss were returned to the original paired operant condition in which both keys were simultaneously available and produced points on their respective schedules. In the extinction procedures the key placed on extinction was freely available to $\mathrm{S}$ but did not produce points on S's counter; the key not on extinction was also freely available to $S$ and did produce points on S's counter. In the contingent case, the higher rate key in the paired operant sessions (the CRF key) was made contingent upon the low rate key (the FR5 key); the CRF key could produce points on S's counter only during the $10 \mathrm{sec}$. interval that immediately followed completion of each FR5 requirement.

\section{Results}

Rate of key pressing for both Ss in the initial paired operant, in CRF extinction, in FR extinction, in the contingent case, and in the final paired operant are shown in Fig. 1. Rate of responding on the CRF key clearly exceeds rate of responding on the FR key for both Ss in the paired operant sessions. Extinction of the CRF key had the effect of an immediate increase in rate of responding on the FR key and a simultaneous reduction of CRF key rate for both Ss. Extinction of the FR key reduced the FR key response rate for both Ss, more dramatically for the male who had a higher paired operant FR key rate than for the female. Rate of responding on the CRF key was largely unaffected by the FR extinction procedure. In the final paired operant, rates of responding on both keys for both Ss show good recoverability of the baseline response rates that were obtained in the original paired operant.

The reinforcing effect of the higher rate CRF key is evident in the increased rate of FR key responding in 


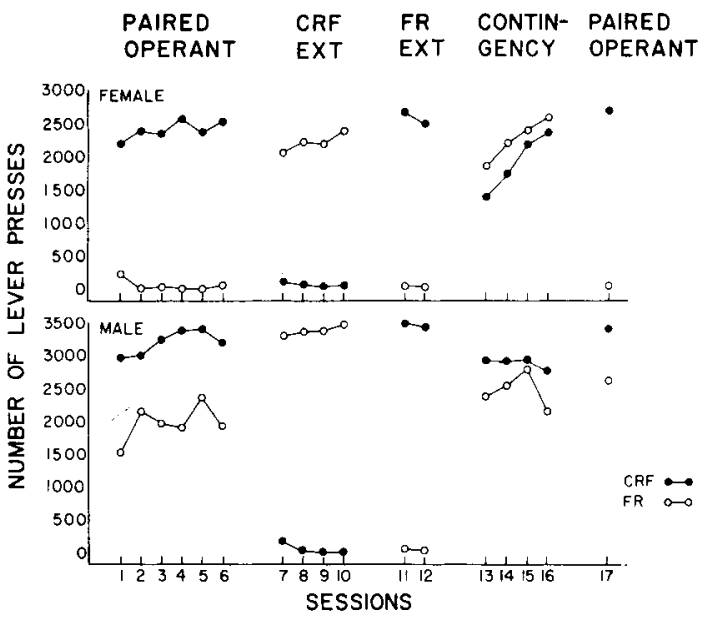

Fig. 1. Total number of CRF and FR lever presses per session for each of the five experimental conditions for the female $S$ (upper panel) and the male $S$ (lower panel).

the contingent case. The magnitude of the reinforcement effect was assessed by a procedure originally suggested by Schaeffer (1962); namely, subtracting mean rate of the instrumental response in the paired operant condition from mean rate of the instrumental response in the contingent case. Using this procedure, the mean reinforced increments in FR key pressing associated with the contingency were 2292.2 and 481.3 for the female $S$ and the male $\mathrm{S}$, respectively.

Differing rates of responding are evident for the two Ss in the contingent case; the male $S$, who had a high rate of FR key responding in the paired operant, reached asymptote on the third day of the contingency, while the female $S$, who had a low rate of FR key responding in the paired operant, was still increasing her rate after four days of the contingency.

\section{Discussion}

The prediction has often been made in private discussions that Premack's reinforcement theory would not hold for cases in which a given reinforcer was contingent upon two similar responses with different schedule requirements. The rationale for this prediction is as follows: first, for a specific reinforcer, and a specific instrumental response, FR schedules, when tested separately from CRF schedules, produce higher response rates than do CRF schedules (Skinner, 1938); second, any attempt to make the higher rate response (the FR response) contingent upon the lower rate response (the CRF response) would fail to reinforce the lower rate $\mathrm{CRF}$ response, since the lower rate response would itself be reinforced when it occurred, the rein- forcement being sufficient to preclude any occurrence of the contingent FR response. It must be pointed out that this prediction, and the rationale underlying it, is based upon a misunderstanding of Premack's basic assumption: namely, that response rates must be determined in the case where both responses are simultaneously available to the $S$ so that $S$ may choose freely between them. Premack's theory makes no assumptions about the reinforcement effect where each response is tested separately, and the $S$ has no choice of responses. Furthermore, as was evident in the present study, when two responses with different schedule requirements which produce the same reinforcer are concurrently available, the $S$ chooses the response with the lower schedule requirement more frequently than the response with the higher requirement. Thus, the ordinal relation between CRF and FR5 response rates when tested concurrently is obviously the inverse of the traditional case where CRF and FR5 are tested separately. Nonetheless, the present study provided a test under conditions for which it has been assumed the differential rate reinforcement generalization would not hold. The present results provide further confirmation for the general applicability of Premack's reinforcement theory. The differential rates of approach to asymptotic contingent responding that were found in the present study also support the contention that rate of responding in the contingent case is partly influenced by the independent rate of the instrumental response, and is not entirely attributable to the independent rate of the reinforcing response (Premack, 1965; Schaeffer, 1965).

\section{References}

Premack, D. Toward empirical behavior laws: I. Positive reinforcement. Psychol. Rev., 1959, 66, 219-233.

Premack, D. Predicting instrumental performance from the independent rate of the contingent response. J. exp. Psychol., 1961 61, 163-171.

Premack, D. Reversibility of the reinforcement relation. Science, 1962, 136, 255-257.

Premack, D. Rate differential reinforcement in monkey manipulation. J. exp. Anal. Beh., 1963, 81-89.

Premack, D. Reinforcement theory. In Nebraska Symposium on Motivation, 1965. Lincoln: University Nebraska Press, 1965.

Schaeffer, R. W. Contributions of the operant level of the instrumental response to the reinforcement relation. Unpublished doctoral dissertation, University of Missouri, 1962.

Schaeffer, R. W. The reinforcement relation as a function of instrumental response base rate. J. exp. Psychol., 1965, 69, 419425 .

Skinner, B. F. The behavior of organisms. New York: AppletonCentury, 1938.

\section{Note}

1. Supported in whole by Public Health Service Research Grant $\mathrm{MH} 08775$, from the National Institute of Mental Health. 